Article

\title{
Design, Synthesis, and Antifungal Activity of Novel Aryl-1,2,3-Triazole- $\beta$-Carboline Hybrids
}

\author{
Xin-Yu Huo ${ }^{1,+}{ }^{,}$Liang Guo ${ }^{1,+}$, Xiao-Fei Chen ${ }^{1}$, Yue-Ting Zhou ${ }^{2}$, Jie Zhang ${ }^{1, *}$, \\ Xiao-Qiang Han ${ }^{2, *}$ (iD) and Bin Dai ${ }^{1, *}$ \\ 1 School of Chemistry and Chemical Engineering, Key Laboratory for Green Processing of Chemical \\ Engineering of Xinjiang Bingtuan, Shihezi University, Shihezi 832003, China; \\ shz_hxy1993@sina.com (X.-Y.H.); guoliang_xj@163.com (L.G.); mykoob@163.com (X.-F.C.) \\ 2 Key Laboratory at Universities of Xinjiang Uygur Autonomous Region for Oasis Agricultural Pest \\ Management and Plant Protection Resource Utilization, College of Agricultural, Shihezi University, \\ Shihezi 832003, China; zhouyueting@stu.shzu.edu.cn \\ * Correspondence: zhangjie-xj@163.com (J.Z.); hanshz@shzu.edu.cn (X.-Q.H.); db_tea@shzu.edu.cn (B.D.); \\ Tel.: +86-993-205-7215 (J.Z.); +86-993-205-8060 (X.-Q.H.); +86-993-205-8176 (B.D.) \\ + These authors contributed equally to this work.
}

Received: 4 May 2018; Accepted: 31 May 2018; Published: 4 June 2018

\begin{abstract}
The copper catalytic azide and terminal alkyne cycloaddition reaction, namely "click chemistry", gives a new and convenient way to create 1,4-disubstitutd-1,2,3-triazoles. In this work, 2-pyrrolecarbaldiminato- $\mathrm{Cu}$ (II) complexes were established as efficient catalysts for the three-component 1,3-dipolar cycloaddition reaction of arylboronic acid and sodium azide $\left(\mathrm{NaN}_{3}\right)$ with terminal alkynes in ethanol at room temperature to $50^{\circ} \mathrm{C}, 1,4$-disubstituted 1,2,3-triazoles were synthesized. Following the optimized protocol, two series of new aryl-1,2,3-triazole- $\beta$-carboline hybrids have been designed and synthesized, and the chemical structures were characterized by ${ }^{1} \mathrm{H}$ NMR, ${ }^{13} \mathrm{C}$ NMR, and high-resolution mass spectrometry (HRMS). All of the target compounds were evaluated in vitro for their antifungal activity against Rhizoctorzia solani, Fusarium oxysporum, Botrytis cinerea Pers., sunflower sclerotinia rot, and rape sclerotinia rot by mycelia growth inhibition assay at $50 \mu \mathrm{g} / \mathrm{mL}$. The antifungal evaluation of the novel hybrids showed that, among the tested compounds, $\mathbf{5 a}, \mathbf{5 b}, \mathbf{5 c}$, and $\mathbf{9 b}$ showed good antifungal activity against sunflower sclerotinia rot. Specifically, compound $\mathbf{9 b}$ also exhibited high broad-spectrum fungicidal against all the tested fungi with inhibition rates of $58.3 \%, 18.52 \%, 63.07 \%, 84.47 \%$, and $81.23 \%$. However, for $F$. oxysporum, all the target compounds showed no in vitro antifungal activities with an inhibition rate lower than $20 \%$. These results provide an encouraging framework that could lead to the development of potent novel antifungal agents.
\end{abstract}

Keywords: $\beta$-carboline; 1,2,3-trizole; antifungal activity; structure-activity relationships

\section{Introduction}

Plant pathogenic microorganisms could infect crops and cause local or whole plant disease, which leads to significant economic losses [1]. In recent years, the potential impact of synthetic pesticides on the environment and human health has been of great concern, which highlights the need for environmentally-friendly pesticides to protect crops from insect infestation [2]. Therefore, plant-derived extracts and their bioactive natural compounds have been considered bio-rational alternatives [3]. Additionally, further modification and structural optimization of novel insecticides leading from the plant origin have recently been important methods for the research and development of new pesticides [4]. Harmine, harman, and harmol, belonging to the $\beta$-carboline alkaloid class, are 
present in medicinal plants, such as Peganum harmala L. [5]. The reported biological applications of $\beta$-carboline alkaloids include sedative and anxiolytic [6], antitumor [7,8], antimalarial [8], antiparasitic [9], anti-HIV [10] agents, and other pharmacological activities. As for pest management, the extracts of Peganum harmala L. plant species containing a mixture of harmine, harmaline, and norharman, as well as their derivatives, had been proven to have excellent insecticidal, fungicidal, and plant growth regulatory properties [11-17]. In our previous work [18], we found that 9-fluorosubstituted-harmine displayed higher fungicidal activities against Rhizoctonia solani, Rape sclerotinia rot, and Alternaria kikuchiana Tanaka.

1,2,3-Triazole and its derivatives as an important class of nitrogen-containing aromatic heterocyclic compounds have attracted a great deal of interest due to their diverse biological activities, such as anticancer $[19,20]$ and antifungal [20] activities, and other properties [21,22]. Meanwhile, the 1,2,3-triazole moiety is stable with regard to metabolic degradation, and capable of hydrogen bonding, which could be favorable in binding of biomolecular targets and increasing solubility [23]. Moreover, 1,2,3-triazoles can be attractive as linker units, which could connect two pharmacophores to give an innovative bifunctional drug, and have become increasingly useful and important in constructing bioactive molecules [24,25].

Accordingly, in an attempt to improve activity of $\beta$-carboline derivatives, in this paper, we synthesized two series of novel aryl-1,2,3-triazole- $\beta$-carboline hybrids (see Figure 1). Their antifungal activities were evaluated in vitro.

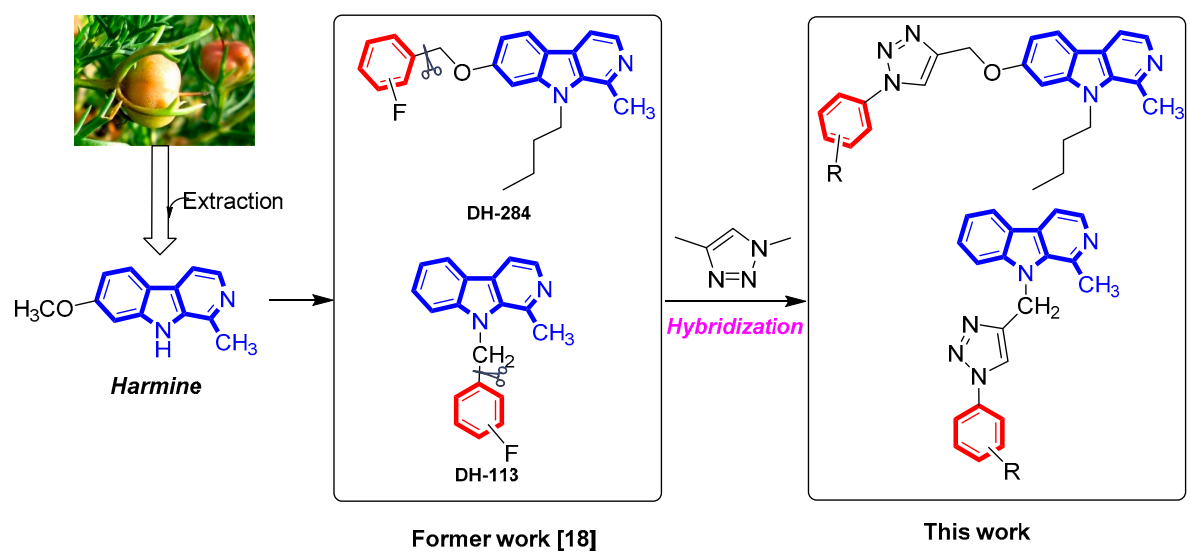

Figure 1. Designed strategy of aryl-1,2,3-triazole- $\beta$-carboline hybrids.

\section{Results and Discussion}

\subsection{Chemistry}

The synthesis of the desired key intermediate 1-methyl-9-(prop-2-yn-1-yl)- $\beta$-carboline (3) was performed in three steps starting from L-tryptophan, which was outlined in Scheme 1. The synthetic step involved the Pictet-Spengler condensation [6], and was followed by oxidation and decarboxylation to afford the intermediate 1-methyl- $\beta$-carboline (2). In the next step, the $N^{9}$-alkylated of compound 2 was prepared by the action of sodium hydride $(\mathrm{NaH})$ in anhydrous $\mathrm{N}, \mathrm{N}$-dimethylformamide (DMF) followed by addition of propargyl bromide to afford compound 3, which incorporates an alkynyl group required for click chemistry.

A number of synthetic methodologies $[19,26,27]$ are available in the literature for the synthesis of 1,2,3-triazole. In our previous investigation [28,29], we have found that 2-pyrrolecarbaldiminato-Cu(II) complexes are efficient catalysts, which affords the 1-benzyl-1,2,3-triazoles in good yields. In order to improve the selectivity of the reaction, we have studied the reaction conditions by screening various catalysts. Initially, the cycloaddition reaction between phenylboronic acid, $\mathrm{NaN}_{3}$, and 1-methyl-9-propargyl- $\beta$-carboline (3) was selected as a model reaction to investigate the catalytic 
activity of four different 2-pyrrolecarbaldiminato- $\mathrm{Cu}(\mathrm{II})$ complexes, and the results are summarized in Table 1. It was found that the azidonation reaction of phenylboronic acid with $\mathrm{NaN}_{3}$ proceeded smoothly within $8 \mathrm{~h}$ in the presence of the four $\mathrm{Cu}$ (II) complexes with $1 \mathrm{~mol} \%$ loading. Subsequently, we added intermediate 3 to the reaction mixture, and the solution was heated at $50{ }^{\circ} \mathrm{C}$ for $2 \mathrm{~h}$. The click cyclization reaction was completed to give the 1,4-disubstituted 1,2,3-triazoles in the yields of $69 \%$ to $84 \%$, and $\mathrm{Cu}(\mathrm{II})$-complex $\mathrm{L}_{1}$ was found to be the best (Entries 1-4). The control experiment indicated that the reaction could not occur without the $\mathrm{Cu}(\mathrm{II})$-complex (Entry 5). When the amount of the $\mathrm{Cu}(\mathrm{II})$-complex, $\mathrm{L}_{1}$, was reduced from $1 \mathrm{~mol} \%$ to $0.5 \mathrm{~mol} \%$, it resulted in a lower yield (Entry 6). Therefore, the optimal conditions for aryl-1,2,3-triazole- $\beta$-carboline hybrid synthesis involves the use of $1 \mathrm{~mol} \% \mathrm{Cu}(\mathrm{II})$-complex $\mathrm{L}_{1}$ as the catalyst. and ethanol as the solvent.

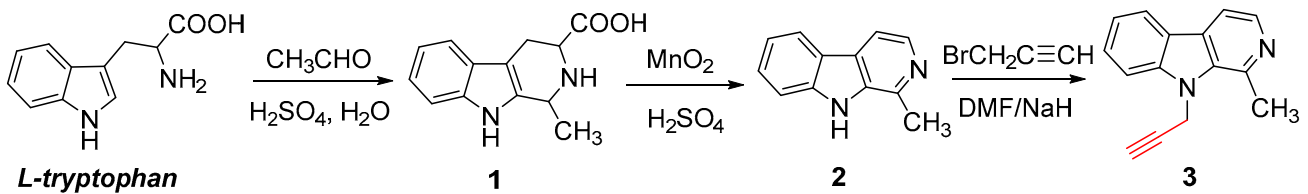

Scheme 1. Synthesis of the key intermediate 3.

Table 1. Cu(II)-complex-catalyzed one-pot synthesis of aryl-1,2,3-triazole- $\beta$-carboline hybrids from phenylboronic acid in ethanol: optimization of the catalytic conditions.

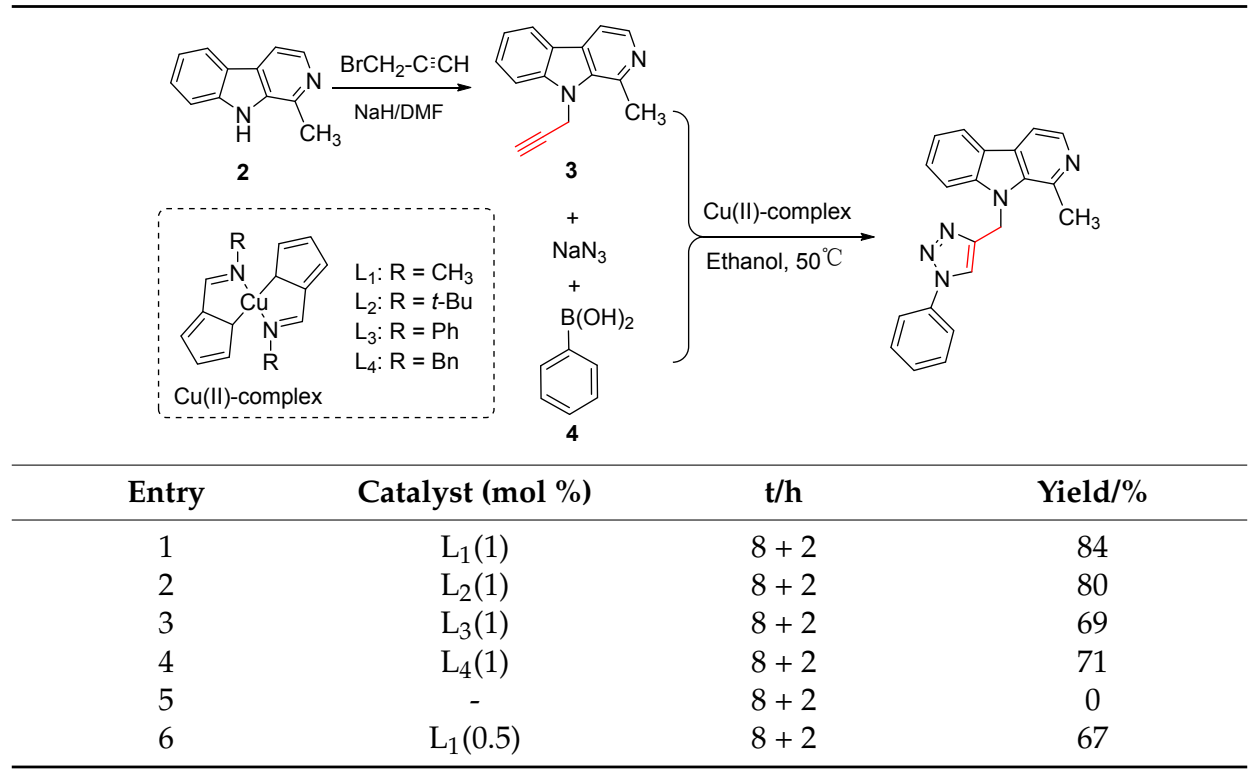

The generality of the optimized reaction condition was studied with a wide range of substrates, using various substituted phenylboronic acid bearing electron-withdrawing and electron-donating substituents, $\mathrm{NaN}_{3}$, and $1 \mathrm{~mol} \% \mathrm{Cu}$ (II)-complex $\mathrm{L}_{1}$ with 1-methyl-9-propargyl- $\beta$-carboline 3 to afford 9-(1,2,3-triazolyl)- $\beta$-carboline hybrids $\mathbf{5 a}-\mathbf{k}$, which are shown in Scheme 2 . The synthetic routes of novel 7-(1,2,3-triazolyl)- $\beta$-carboline hybrids 9a-f are outlined in Scheme 3 . The $N^{9}$-alkylated harmine derivative 6 was prepared according to the synthetic protocol described by our group [30]. The preparation of compound 7 followed a common synthetic scheme and was characterized by demethylation of compound 6 using hydrobromic acid and acetic acid as the reaction solvent. Compound 8 , bearing alkoxy in postion-7 of $\beta$-carboline core, was synthesized from compound 7 by the action of $\mathrm{NaH}$ in dry DMF followed by addition of propargyl bromide in $81 \%$ yield. Lastly, the synthesis of compounds $\mathbf{9 a}-\mathbf{f}$ was carried out following the general procedure for the synthesis of compounds $\mathbf{5 a - k}$. All structures of the final products were determined by ${ }^{1} \mathrm{H}$ NMR, ${ }^{13} \mathrm{C}$ NMR (see Supplementary Materials), and HRMS. 

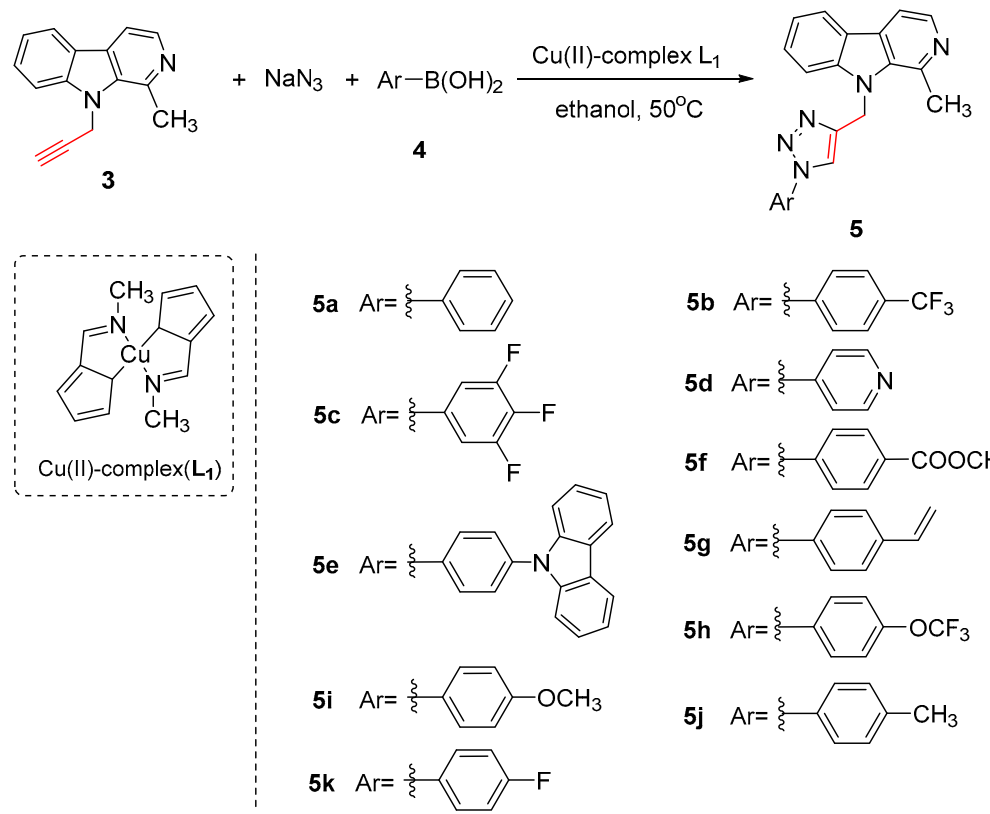

$5 d \quad A r=\{-N$

5f $\mathrm{Ar}=\xi-\mathrm{COOCH}_{2} \mathrm{CH}_{3}$

$5 \mathrm{~g} \quad \mathrm{Ar}=\xi$

5h $\mathrm{Ar}=\left\{-\mathrm{OCF}_{3}\right.$

5j $\quad \mathrm{Ar}=\xi-\mathrm{CH}_{3}$

Scheme 2. Synthesis of the 9-(1,2,3-triazolyl)- $\beta$-carboline hybrids $5 \mathbf{a}-\mathbf{k}$.
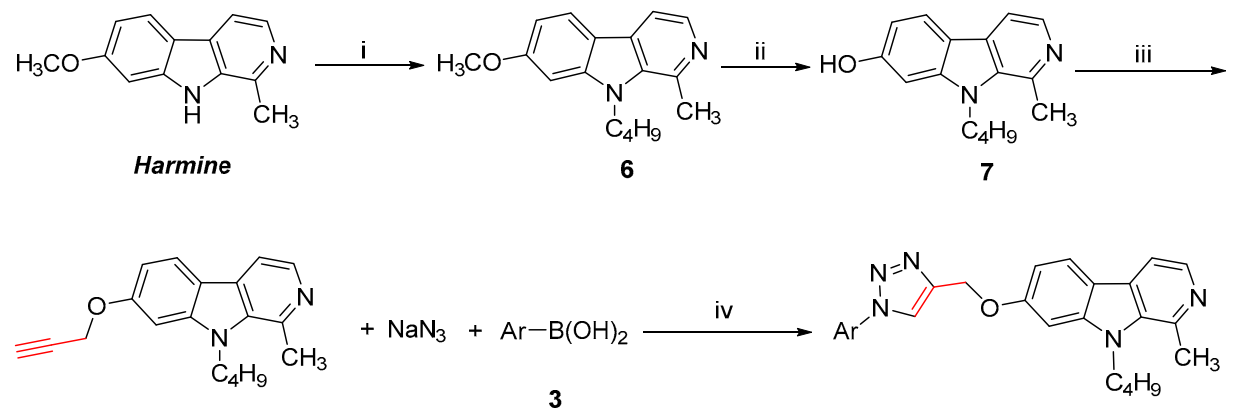

8

3

9
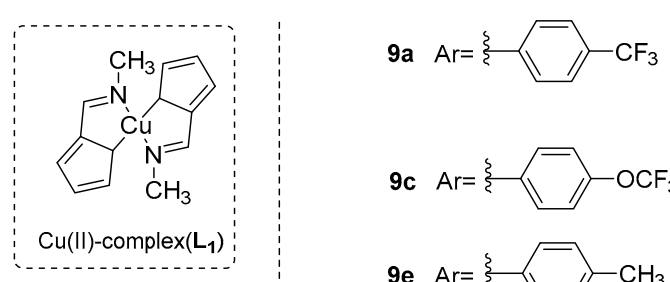

9b $A r=$

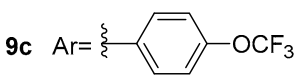

9d $\mathrm{Ar}=\xi-\mathrm{OCH}_{3}$

9e $\quad \mathrm{Ar}=\xi-\mathrm{CH}_{3}$

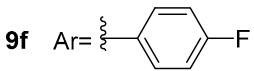

Scheme 3. Synthesis of the 7-(1,2,3-triazolyl)- $\beta$-carboline hybrids 9a-f. Reagents and conditions: (i) DMF, NaH, $n$-iodobutane, stirred at RT; (ii) $\mathrm{HBr}, \mathrm{HOAc}$, reflux (iii) DMF, NaH, 3-bromopropyne, stirred at RT; (iv) $\mathrm{Cu}$ (II)-complex $\mathrm{L}_{1}$, ethanol, $50{ }^{\circ} \mathrm{C}$.

\subsection{Fungicidal Activities}

From the synthetic route mentioned above, we obtained two series of novel aryl-1,2,3-triazole- $\beta$ carboline hybrids $\mathbf{5 a}-\mathbf{k}, \mathbf{9 a}-\mathbf{f}$. These compounds were evaluated in a series of fungicidal tests in vitro against a range of phytopathogenic species including R. solani, Fusarium oxysporum, Botrytis cinerea Pers., sunflower sclerotinia rot, and rape sclerotinia rot. The activity results obtained as an inhibition rate are summarized in Table 2.

Generally, at $50 \mu \mathrm{g} / \mathrm{mL}$, the target compounds exhibited different levels of antifungal activity against these five tested fungi. Compared with that of the commercial fungicide carbendazim and azoxystrobin, these compounds have exhibited a significant inhibitory effect against sunflower 
sclerotinia rot (SCR) in which compounds 5a (Ar = phenyl), 5b (Ar = 4-trifluoromethylphenyl), 5c (Ar = 3,4,5-trifluorophenyl), and 9b (Ar = 3,4,5-trifluorophenyl) had inhibitory rates of 85.04\%, 86.93\%, $85.98 \%$, and $84.47 \%$, respectively, which displayed comparable antifungal activity than that of the positive control, with an inhibition rate of $89.77 \%$ and $88.07 \%$. In addition, compounds $5 \mathbf{d}-\mathbf{g}, \mathbf{5 i}-\mathbf{k}, \mathbf{9 c}-\mathbf{d}$, and $9 f$ displayed moderate activity, with an inhibition rate ranging from $50 \%$ to $80 \%$. For F. oxysporum, all the target compounds showed inactive in vitro antifungal activities with an inhibition rate lower than 20\%. Similarly, for R. solani, the compounds showed weak antifungal activities with an inhibition rate ranging from $20 \%$ to $50 \%$, except for 9 b, which exhibited moderate activity with an inhibition rate of $58.30 \%$. However, it was not as clear as the one drawn from the RSR data. Some of the compounds exhibited significant activities in vitro toward RSR in which the compound $\mathbf{9 b}$ had control efficacy rates of $81.23 \%$ and most of them showed weak to moderate activity. Of all aryl-1,2,3-triazole- $\beta$-carboline hybrids, compound $\mathbf{9 b}$ displayed as broad a fungicidal spectrum as azoxystrobin and carbendazim against these phytopathogens.

Table 2. Fungicidal activities of compounds $\mathbf{5 a}-\mathbf{k}, \mathbf{9 a}-\mathbf{f}$ against four kinds of fungi $(50 \mu \mathrm{g} / \mathrm{mL})^{\mathrm{a}}$.

\begin{tabular}{|c|c|c|c|c|c|c|}
\hline \multirow{2}{*}{ Componds } & \multicolumn{5}{|c|}{ Inhibition Ratio (\%) $b$} & \multirow{2}{*}{$C \log P$} \\
\hline & RS & FO & BCP & SCR & RSR & \\
\hline $5 a$ & 35.57 & -2.60 & 51.14 & 85.04 & 22.22 & 4.251 \\
\hline $5 b$ & 30.68 & -0.28 & 38.64 & 86.93 & 0.00 & 5.435 \\
\hline $5 c$ & 33.52 & 0.65 & 34.85 & 85.98 & 53.26 & 4.788 \\
\hline $5 d$ & 25.23 & -1.67 & 36.36 & 58.14 & 0.00 & 3.468 \\
\hline $5 e$ & 28.07 & -2.37 & 15.53 & 71.78 & 17.05 & 8.437 \\
\hline $5 f$ & 27.84 & 1.11 & 14.02 & 73.48 & 35.44 & 5.063 \\
\hline $5 g$ & 33.52 & -0.28 & 15.91 & 67.99 & 26.05 & 4.976 \\
\hline $5 h$ & 35.80 & -2.60 & 19.70 & 44.70 & 55.36 & 5.588 \\
\hline $5 \mathbf{i}$ & 30.45 & 1.81 & 16.29 & 65.34 & 19.54 & 4.479 \\
\hline $5 \mathbf{j}$ & 34.09 & -3.76 & 7.39 & 67.80 & 35.25 & 4.750 \\
\hline $5 k$ & 34.09 & 0.19 & 5.11 & 70.08 & 0.00 & 4.566 \\
\hline $9 a$ & 36.59 & -0.97 & 12.69 & 40.53 & 10.76 & 7.306 \\
\hline $9 b$ & 58.30 & 18.52 & 63.07 & 84.47 & 81.23 & 6.659 \\
\hline $9 c$ & 39.20 & 7.85 & 16.86 & 79.31 & 52.11 & 7.459 \\
\hline $9 d$ & 28.75 & 2.27 & 63.45 & 76.70 & 47.31 & 6.350 \\
\hline $9 e$ & 47.72 & -7.24 & 28.03 & 44.51 & 28.91 & 6.621 \\
\hline 9f & 35.34 & 0.65 & 24.43 & 75.57 & 19.28 & 6.437 \\
\hline carbendazim & 81.82 & 70.98 & 88.07 & 89.77 & 100 & \\
\hline azoxystrobin & 54.55 & 51.25 & 83.71 & 88.07 & 88.51 & \\
\hline
\end{tabular}

${ }^{a}$ RS, R. solani; FO, F. oxysporum; BCP, B. cinerea Pers.; SCR, sunflower sclerotinia rot; RSR, rape sclerotinia rot. The data in bold are used to emphasize that these compounds showed good activity. ${ }^{b}$ significant inhibitory effect: inhibitory rate $\geq 80 \%$, moderate: inhibition rate ranges from $50 \%$ to $80 \%$, weak: inhibition rate ranges from $20 \%$ to $50 \%$. ${ }^{c} C \log P$ represent the calculated $n$-octanol/water partition coefficient (log Pow), and the values produced by Chemdraw software.

\section{Materials and Methods}

\subsection{General Information}

All the reactions were monitored by TLC on silica gel F254 plates (Qingdao Haiyang Inc., Qingdao, China) for detection of the spot. Column chromatography was performed with silica gel (200-300 mesh). NMR spectra were recorded at room temperature on a Bruker Avance III HD 400 instrument at $400 \mathrm{MHz}$ for ${ }^{1} \mathrm{H}$ NMR and $100 \mathrm{MHz}$ for ${ }^{13} \mathrm{C}$ NMR (Bruker Company, Bremen, Gemany). CDCl $3, \mathrm{DMSO} d_{6}$, Methanol- $d_{4}$ or Pyridine- $d_{5}$ was used as the solvent and TMS as the internal standard. High-resolution mass spectrometry (HRMS) were measured on Bruker ultrafleXtreme MALDI-TOF/TOF-MS and HCCA (alpha-cyano-4-hydroxycinnamic acid) is used as matrix. 
All solvents were purified and dried using standard methods prior to use. The following intermediates, 1-methyl- $\beta$-carboline 2 [31], 7-methoxy-9-n-butyl-1-methyl- $\beta$-carboline 6 [30] and 9-n-butyl-1-methyl- $\beta$-carboline-7-ol 7 [32] were synthesized according to published procedures.

\subsection{Synthesis of 1-Methyl-9-(prop-2-yn-1-yl)- $\beta$-carboline (3)}

A mixture of 1-methyl- $\beta$-carboline $(2,1.82 \mathrm{~g}, 10 \mathrm{mmol})$ and anhydrous DMF $(60 \mathrm{~mL})$ was stirred at room temperature for $0.5 \mathrm{~h}$, and then $95 \% \mathrm{NaH}(0.37 \mathrm{~g}, 15 \mathrm{mmol})$ and 3-bromopropyne $(1.8 \mathrm{~g}, 15$ $\mathrm{mmol}$ ) were added. The mixture was stirred at room temperature for $15-45 \mathrm{~min}$. After completion of the reaction as indicated by TLC, the solution was poured into $\mathrm{H}_{2} \mathrm{O}(150 \mathrm{~mL})$, and extracted with ethyl acetate. The organic phase was washed with water and brine, then dried over anhydrous sodium sulfate, filtered, and evaporated. The resulting oil was crystallized from ethyl ether. White crystals of 3 were obtained $(1.93 \mathrm{~g}, 88 \%) .{ }^{1} \mathrm{H}$ NMR $\left(400 \mathrm{MHz}, \mathrm{DMSO}-d_{6}\right) \delta: 8.38-8.18(\mathrm{~m}, 2 \mathrm{H}), 8.01(\mathrm{~s}, 1 \mathrm{H}), 7.81(\mathrm{~d}$, $J=7.6 \mathrm{~Hz}, 1 \mathrm{H}), 7.64(\mathrm{t}, J=7.6 \mathrm{~Hz}, 1 \mathrm{H}), 7.32(\mathrm{t}, J=6.8 \mathrm{~Hz}, 1 \mathrm{H}), 5.48(\mathrm{~s}, 2 \mathrm{H}), 3.39(\mathrm{~d}, J=2.0 \mathrm{~Hz}, 1 \mathrm{H}), 3.09$ $(\mathrm{s}, 3 \mathrm{H}) .{ }^{13} \mathrm{C}$ NMR $\left(100 \mathrm{MHz}, \mathrm{DMSO}-d_{6}\right) \delta: 142.22,141.34,138.80,134.74,129.05,128.82,122.07,121.34$, $120.62,113.49,110.86,80.58,76.10,34.68,23.10$.

\subsection{Synthesis of 9-Butyl-1-Methyl-7-(Prop-2-yn-1-yloxy)- $\beta$-Carboline (8)}

Prepared by the same procedure as compound 3 from 7 (2.54 g, $10 \mathrm{mmol})$ and 3-bromopropyne $(1.8 \mathrm{~g}, 15 \mathrm{mmol})$. White crystals of 8 were obtained $(2.37 \mathrm{~g}, 81 \%) .{ }^{1} \mathrm{H} \mathrm{NMR}\left(400 \mathrm{MHz}, \mathrm{CDCl}_{3}\right) \delta: 8.29(\mathrm{~d}$, $J=5.2 \mathrm{~Hz}, 1 \mathrm{H}), 7.99(\mathrm{dd}, J=8.1,2.0 \mathrm{~Hz}, 1 \mathrm{H}), 7.73(\mathrm{~d}, J=5.2 \mathrm{~Hz}, 1 \mathrm{H}), 6.99(\mathrm{t}, J=2.0 \mathrm{~Hz}, 1 \mathrm{H}), 6.95-6.92$ $(\mathrm{m}, 1 \mathrm{H}), 4.84(\mathrm{dd}, J=2.4,1.2 \mathrm{~Hz}, 2 \mathrm{H}), 4.46(\mathrm{t}, J=8.0 \mathrm{~Hz}, 2 \mathrm{H}), 3.02(\mathrm{~s}, 3 \mathrm{H}), 2.57(\mathrm{t}, J=2.4 \mathrm{~Hz}, 1 \mathrm{H})$, 1.86-1.77 (m, 2H), 1.50-1.40 (m, 2H), $0.98(\mathrm{t}, J=7.2 \mathrm{~Hz}, 3 \mathrm{H}) .{ }^{13} \mathrm{C}$ NMR $\left(100 \mathrm{MHz}, \mathrm{CDCl}_{3}\right) \delta: 158.55$, $142.81,140.73,138.28,135.44,129.17,122.39,115.97,112.35,108.92,95.16,78.5,75.79,56.35,44.77,32.77$, $23.47,20.23,13.92$.

\subsection{General Procedure for the Synthesis of 1,2,3-Triazolyl- $\beta$-Carboline Hybrids (5 and 9)}

A $50 \mathrm{~mL}$ Schlenk tube was charged with $\mathrm{Cu}(\mathrm{II})$-complex $\mathrm{L}_{1}(0.025 \mathrm{mmol})$, arylboronic acid $(5 \mathrm{mmol}), \mathrm{NaN}_{3}(6 \mathrm{mmol})$ and dry alcohol $(30 \mathrm{~mL})$. The mixture was stirred at $30{ }^{\circ} \mathrm{C}$ and monitored by TLC until the arylboronic acid was consumed. Compound 3 or 8 (2.5 mmol) was added, and the solution was continuously heated at $50{ }^{\circ} \mathrm{C}$ for $2 \mathrm{~h}$. After completion of the reaction, water was added to the reaction mixture, and the compound was extracted with ethyl acetate $(3 \times 100 \mathrm{~mL})$. The organic phase was washed with water and brine, dried over anhydrous $\mathrm{Na}_{2} \mathrm{SO}_{4}$, and the solvent was removed under reduced pressure. The crude product was purified by flash column chromatograph on silica gel (ethyl acetate/petroleum ether as the eluent) to obtain the target products.

1-Methyl-9-((1-phenyl-1H-1,2,3-triazol-4-yl)methyl)- $\beta$-carboline (5a): White crystals $(0.71 g, 84 \%)$ were obtained. ${ }^{1} \mathrm{H}$ NMR $\left(400 \mathrm{MHz}, \mathrm{DMSO}-d_{6}\right) \delta: 8.79(\mathrm{~s}, 1 \mathrm{H}), 8.24-8.27(\mathrm{~m}, 2 \mathrm{H}), 8.02(\mathrm{~d}, J=5.2 \mathrm{~Hz}, 1 \mathrm{H})$, 7.81-7.88 (m, 3H), 7.58-7.62 (m, 1H), 7.52-7.56 (m, 2H), 7.43-7.47 (m, 1H), 7.29 (t, J = 7.2 Hz, 1H), 5.99 (s, 2H), 3.13 (s, 3H). ${ }^{13} \mathrm{C}$ NMR $\left(100 \mathrm{MHz}, \mathrm{DMSO}-d_{6}\right) \delta: 145.53,142.30,141.46,138.38,136.89,135.09$, $130.25,129.16,128.67,128.65,121.94,121.67,121.38,120.57,120.34,113.46,111.18,39.92,23.89$. HRMS calcd for $\mathrm{C}_{21} \mathrm{H}_{18} \mathrm{~N}_{5}[\mathrm{M}+\mathrm{H}]^{+}$340.1557, found 340.1569 .

1-Methyl-9-((1-(4-(trifluoromethyl)phenyl)-1H-1,2,3-triazol-4-yl)methyl)- $\beta$-carboline (5b): Slightly brown crystals $(0.87 \mathrm{~g}, 86 \%)$ were obtained. ${ }^{1} \mathrm{H}$ NMR $\left(400 \mathrm{MHz}, \mathrm{DMSO}-d_{6}\right) \delta: 8.93(\mathrm{~s}, 1 \mathrm{H}), 8.26(\mathrm{~d}, J=7.6 \mathrm{~Hz}$, $2 \mathrm{H}), 8.10(\mathrm{~d}, J=8.4 \mathrm{~Hz}, 2 \mathrm{H}), 8.03(\mathrm{~d}, J=5.2 \mathrm{~Hz}, 1 \mathrm{H}), 7.93(\mathrm{~d}, J=8.8 \mathrm{~Hz}, 2 \mathrm{H}), 7.86(\mathrm{~d}, J=8.4 \mathrm{~Hz}, 1 \mathrm{H})$, $7.58-7.63(\mathrm{~m}, 1 \mathrm{H}), 7.30(\mathrm{t}, J=8.0 \mathrm{~Hz}, 1 \mathrm{H}), 6.02(\mathrm{~s}, 2 \mathrm{H}), 3.12(\mathrm{~s}, 3 \mathrm{H}) .{ }^{13} \mathrm{C}$ NMR $\left(100 \mathrm{MHz}, \mathrm{DMSO}-d_{6}\right) \delta$ : $145.99,142.27,141.46,135.09,139.66$ (q, $J=1.4 \mathrm{~Hz}), 138.41,128.96$ (q, $J=32.1 \mathrm{~Hz}), 128.71,128.63,127.55$ $(\mathrm{q}, J=3.7 \mathrm{~Hz}), 124.24(\mathrm{q}, J=270.5 \mathrm{~Hz}), 121.95,121.39,121.03,120.37,113.47,111.15,23.86 .{ }^{19} \mathrm{~F} \mathrm{NMR}$ $\left(376 \mathrm{MHz}, \mathrm{DMSO}-d_{6}\right) \delta$ : -61.04 . HRMS calcd for $\mathrm{C}_{22} \mathrm{H}_{17} \mathrm{~F}_{3} \mathrm{~N}_{5}[\mathrm{M}+\mathrm{H}]^{+}$408.1431, found 408.1422. 
1-Methyl-9-((1-(3,4,5-trifluorophenyl)-1H-1,2,3-triazol-4-yl)methyl)- $\beta$-carboline (5c): Slightly brown crystals $(0.79 \mathrm{~g}, 80 \%)$ were obtained. ${ }^{1} \mathrm{H}$ NMR $\left(400 \mathrm{MHz}, \mathrm{DMSO}-d_{6}\right) \delta: 8.78(\mathrm{~s}, 1 \mathrm{H}), 8.26(\mathrm{~d}, J=6.0 \mathrm{~Hz}, 2 \mathrm{H})$, $8.04(\mathrm{~d}, J=5.2 \mathrm{~Hz}, 1 \mathrm{H}), 7.98(\mathrm{dd}, J=8.8,6.0 \mathrm{~Hz}, 2 \mathrm{H}), 7.83(\mathrm{~d}, J=8.4 \mathrm{~Hz}, 1 \mathrm{H}), 7.58-7.62(\mathrm{~m}, 1 \mathrm{H}), 7.30(\mathrm{t}$, $J=8.0 \mathrm{~Hz}, 1 \mathrm{H}), 6.01(\mathrm{~s}, 2 \mathrm{H}), 3.09(\mathrm{~s}, 3 \mathrm{H}) .{ }^{13} \mathrm{C} \mathrm{NMR}\left(100 \mathrm{MHz}, \mathrm{DMSO}-d_{6}\right) \delta: 150.43(\mathrm{ddd}, J=240.5,10.1$, $5.6 \mathrm{~Hz}), 145.61,141.61,140.89,138.53(\mathrm{dt}, J=249,14.9 \mathrm{~Hz}), 137.81,134.44,131.92(\mathrm{td}, J=11.5,4.3 \mathrm{~Hz})$, $128.20,128.15,121.52,121.40,120.80,119.84,112.93,110.55,105.71(\mathrm{~m}), 23.18 .{ }^{19} \mathrm{~F}$ NMR $(376 \mathrm{MHz}$, DMSO) $\delta:-132.58(\mathrm{~d}, J=21.8 \mathrm{~Hz}),-161.09(\mathrm{t}, J=21.8 \mathrm{~Hz})$. HRMS calcd for $\mathrm{C}_{21} \mathrm{H}_{15} \mathrm{~F}_{3} \mathrm{~N}_{5}[\mathrm{M}+\mathrm{H}]^{+}$ 394.1274, found 394.1288.

1-Methyl-9-((1-(pyridin-4-yl)-1H-1,2,3-triazol-4-yl)methyl)- $\beta$-carboline (5d): Slightly yellow crystals $(0.61 \mathrm{~g}$, $72 \%$ ) were obtained. ${ }^{1} \mathrm{H}$ NMR $\left(400 \mathrm{MHz}\right.$, Methanol- $\left.d_{4}\right) \delta: 8.66(\mathrm{~d}, J=5.6 \mathrm{~Hz}, 2 \mathrm{H}), 8.55(\mathrm{~s}, 1 \mathrm{H}), 8.23-8.20$ $(\mathrm{m}, 2 \mathrm{H}), 8.01(\mathrm{~d}, J=5.2 \mathrm{~Hz}, 1 \mathrm{H}), 7.89-7.88(\mathrm{~m}, 2 \mathrm{H}), 7.74(\mathrm{dt}, J=8.4,0.8 \mathrm{~Hz}, 1 \mathrm{H}), 7.66-7.62(\mathrm{~m}, 1 \mathrm{H})$, 7.35-7.31 (m, 1H), 6.05 (s, 2H), 3.13 (s, 3H). ${ }^{13} \mathrm{C}$ NMR (100 MHz, Methanol-d 4$) \delta:$ 150.77, 146.20, 143.55, 141.82, 141.61, 137.19, 129.92, 128.60, 121.32, 121.27, 120.33, 120.26, 113.92, 113.08, 109.94, 39.85, 21.46. HRMS calcd for $\mathrm{C}_{20} \mathrm{H}_{17} \mathrm{~N}_{6}[\mathrm{M}+\mathrm{H}]^{+} 341.1509$, found 341.1498.

1-Methyl-9-((1-(4-(9H-carbazol-9-yl)phenyl)-1H-1,2,3-triazol-4-yl)methyl)- $\beta$-carboline (5e): Slightly brown crystals (0.73 g, 58\%) were obtained. ${ }^{1} \mathrm{H}$ NMR $\left(400 \mathrm{MHz}\right.$, Pyridine- $\left.d_{5}\right) \delta: 8.67(\mathrm{~s}, 1 \mathrm{H}), 8.59(\mathrm{~d}, J=5.2 \mathrm{~Hz}$, $1 \mathrm{H}), 8.30-8.26(\mathrm{~m}, 3 \mathrm{H}), 8.05(\mathrm{~d}, J=8.8 \mathrm{~Hz}, 2 \mathrm{H}), 8.00-7.95(\mathrm{~m}, 2 \mathrm{H}), 7.68-7.64(\mathrm{~m}, 5 \mathrm{H}), 7.49(\mathrm{~s}, 2 \mathrm{H})$, 7.41-7.36 (m, 3H), 6.18 (s, 2H), 3.36 (s, 3H). ${ }^{13} \mathrm{C}$ NMR (100 MHz, Pyridine- $\left.d_{5}\right) \delta: 146.26,142.22,141.62$, 140.61, 138.90, 137.55, 135.71, 135.32, 129.16, 128.49, 127.86, 126.45, 123.75, 121.97, 121.91, 121.74, 120.86, $120.66,120.64,120.35,113.11,110.53,109.88,40.75,23.73$. HRMS calcd for $\mathrm{C}_{33} \mathrm{H}_{25} \mathrm{~N}_{6}[\mathrm{M}+\mathrm{H}]^{+} 505.2135$, found 505.2145 .

1-Methyl-9-((1-(4-ethoxycarbonyl)phenyl-1H-1,2,3-triazol-4-yl)methyl)- $\beta$-carboline (5f): Yellow crystals $(0.82$ g, 80\%) were obtained. ${ }^{1} \mathrm{H}$ NMR $\left(400 \mathrm{MHz}\right.$, Methanol- $\left.d_{4}\right) \delta: 8.44(\mathrm{~s}, 1 \mathrm{H}), 8.23-8.20(\mathrm{~m}, 2 \mathrm{H}), 8.16-8.13$ $(\mathrm{m}, 2 \mathrm{H}), 8.02(\mathrm{~d}, J=5.6 \mathrm{~Hz}, 1 \mathrm{H}), 7.92-7.89(\mathrm{~m}, 2 \mathrm{H}), 7.76(\mathrm{~d}, J=8.4 \mathrm{~Hz}, 1 \mathrm{H}), 7.66-7.62(\mathrm{~m}, 1 \mathrm{H}), 7.35-7.31$ $(\mathrm{m}, 1 \mathrm{H}), 6.05(\mathrm{~s}, 2 \mathrm{H}), 4.39(\mathrm{q}, J=7.2 \mathrm{~Hz}, 2 \mathrm{H}), 3.15(\mathrm{~s}, 3 \mathrm{H}), 1.40(\mathrm{t}, J=7.2 \mathrm{~Hz}, 3 \mathrm{H}) .{ }^{13} \mathrm{C} \mathrm{NMR}(100 \mathrm{MHz}$, Methanol- $\left.d_{4}\right) \delta$ : 165.38, 145.83, 141.88, 139.96, 137.15, 137.14, 130.73, 130.43, 129.93, 128.60, 121.33, 121.27, 120.57, 120.24, 119.76, 109.98, 61.08, 39.90, 21.49, 13.13. HRMS calcd for $\mathrm{C}_{24} \mathrm{H}_{22} \mathrm{~N}_{5} \mathrm{O}_{2}[\mathrm{M}+\mathrm{H}]^{+}$ 412.1768 , found 412.1759 .

1-Methyl-9-((1-(4-vinylphenyl)-1H-1,2,3-triazol-4-yl)methyl)- $\beta$-carboline (5g): Brown crystals $(0.81 \mathrm{~g}, 89 \%)$ were obtained. ${ }^{1} \mathrm{H}$ NMR $\left(400 \mathrm{MHz}\right.$, Methanol- $\left.d_{4}\right) \delta: 8.32(\mathrm{~s}, 1 \mathrm{H}), 8.25(\mathrm{~s}, 1 \mathrm{H}), 8.22(\mathrm{~d}, J=8.0 \mathrm{~Hz}, 1 \mathrm{H})$, $8.03(\mathrm{~d}, J=5.2 \mathrm{~Hz}, 1 \mathrm{H}), 7.76(\mathrm{~d}, J=8.4 \mathrm{~Hz}, 1 \mathrm{H}), 7.72-7.70(\mathrm{~m}, 2 \mathrm{H}), 7.66-7.62(\mathrm{~m}, 1 \mathrm{H}), 7.58-7.56(\mathrm{~m}$, 2H), 7.35-7.31 (m, 1H), $6.77(\mathrm{dd}, J=17.6,10.8 \mathrm{~Hz}, 1 \mathrm{H}), 6.04(\mathrm{~s}, 2 \mathrm{H}), 5.85(\mathrm{~d}, J=17.6 \mathrm{~Hz}, 1 \mathrm{H}), 5.32(\mathrm{~d}$, $J=10.8 \mathrm{~Hz}, 1 \mathrm{H}), 3.15$ (s, 3H). ${ }^{13} \mathrm{C}$ NMR $\left(100 \mathrm{MHz}\right.$, Methanol- $\left.d_{4}\right) \delta: 145.47,141.90,138.37,135.99,135.34$, $129.88,128.58,127.05,121.31,121.25,120.39,120.23,120.21,120.20,114.39,109.99,56.92,39.91,16.96$. HRMS calcd for $\mathrm{C}_{23} \mathrm{H}_{20} \mathrm{~N}_{5}[\mathrm{M}+\mathrm{H}]^{+} 366.1713$, found 366.1720 .

1-Methyl-9-((1-(4-(trifluoromethoxy)phenyl)-1H-1,2,3-triazol-4-yl)methyl)- $\beta$-carboline (5h): Slightly brown crystals $(0.84 \mathrm{~g}, 79 \%)$ were obtained. ${ }^{1} \mathrm{H}$ NMR $\left(400 \mathrm{MHz}\right.$, Methanol- $\left.d_{4}\right) \delta: 6.83(\mathrm{~s}, 1 \mathrm{H}), 6.69-6.66(\mathrm{~m}$, $2 \mathrm{H}), 6.47(\mathrm{~d}, J=5.6 \mathrm{~Hz}, 1 \mathrm{H}), 6.35-6.31(\mathrm{~m}, 2 \mathrm{H}), 6.21(\mathrm{~d}, J=8.4 \mathrm{~Hz}, 1 \mathrm{H}), 6.12-6.08(\mathrm{~m}, 1 \mathrm{H}), 5.89(\mathrm{~d}$, $J=8.4 \mathrm{~Hz}, 2 \mathrm{H}), 5.81-5.77(\mathrm{~m}, 1 \mathrm{H}), 4.50(\mathrm{~s}, 2 \mathrm{H}), 1.60(\mathrm{~s}, 3 \mathrm{H}) .{ }^{13} \mathrm{C}$ NMR $\left(100 \mathrm{MHz}\right.$, Methanol- $\left.d_{4}\right) \delta: 144.21$, 140.35, 140.10, 135.61, 133.97, 133.57, 128.40, 127.06, 120.47, 120.40, 119.79, 119.74, 119.18, 118.70, 111.55, 108.44, 38.37, 19.94. ${ }^{19} \mathrm{~F}$ NMR (376 MHz, DMSO- $\left.d_{6}\right) \delta:-59.68$. HRMS calcd for $\mathrm{C}_{22} \mathrm{H}_{17} \mathrm{~F}_{3} \mathrm{~N}_{5} \mathrm{O}[\mathrm{M}+\mathrm{H}]^{+}$ 424.1380 , found 424.1388 .

1-methyl-9-((1-(4-methoxyphenyl)-1H-1,2,3-triazol-4-yl)methyl)- $\beta$-carboline (5i): Brown crystals (0.84 g, 91\%) were obtained. ${ }^{1} \mathrm{H}$ NMR $\left(400 \mathrm{MHz}\right.$, Methanol- $\left.d_{4}\right) \delta: 8.24-8.21(\mathrm{~m}, 3 \mathrm{H}), 8.02(\mathrm{~d}, J=5.2 \mathrm{~Hz}, 1 \mathrm{H})$, $7.75(\mathrm{~d}, J=8.4 \mathrm{~Hz}, 1 \mathrm{H}), 7.66-7.60(\mathrm{~m}, 3 \mathrm{H}), 7.35-7.31(\mathrm{~m}, 1 \mathrm{H}), 7.05-7.01(\mathrm{~m}, 2 \mathrm{H}), 6.02(\mathrm{~s}, 2 \mathrm{H}), 3.83(\mathrm{~s}, 3 \mathrm{H})$, $3.14(\mathrm{~s}, 3 \mathrm{H}) .{ }^{13} \mathrm{C}$ NMR $\left(100 \mathrm{MHz}\right.$, Methanol- $\left.d_{4}\right) \delta: 160.17,145.23,141.89,130.10,129.87,128.58,121.87$, $121.86,121.29,121.24,120.65,120.20,114.39,110.00,54.68,39.90,16.97$. HRMS calcd for $\mathrm{C}_{22} \mathrm{H}_{20} \mathrm{~N}_{5} \mathrm{O}$ $[\mathrm{M}+\mathrm{H}]^{+} 370.1662$, found 370.1669 . 
1-Methyl-9-((1-(p-tolyl)-1H-1,2,3-triazol-4-yl)methyl)- $\beta$-carboline (5j): Slightly brown crystals $(0.76 \mathrm{~g}, 86 \%)$ were obtained. ${ }^{1} \mathrm{H}$ NMR $\left(400 \mathrm{MHz}\right.$, Methanol- $\left.d_{4}\right) \delta: 8.36(\mathrm{~s}, 1 \mathrm{H}), 8.26(\mathrm{~d}, J=8.0 \mathrm{~Hz}, 2 \mathrm{H}), 8.17(\mathrm{~d}$, $J=5.6 \mathrm{~Hz}, 1 \mathrm{H}), 7.81(\mathrm{~d}, J=8.4 \mathrm{~Hz}, 1 \mathrm{H}), 7.72-7.68(\mathrm{~m}, 1 \mathrm{H}), 7.59(\mathrm{~d}, J=8.4 \mathrm{~Hz}, 2 \mathrm{H}), 7.38(\mathrm{t}, J=7.6 \mathrm{~Hz}$, $1 \mathrm{H}), 7.30(\mathrm{~d}, J=8.4 \mathrm{~Hz}, 2 \mathrm{H}), 6.03(\mathrm{~s}, 2 \mathrm{H}), 3.22(\mathrm{~s}, 3 \mathrm{H}), 2.37(\mathrm{~s}, 3 \mathrm{H}) .{ }^{13} \mathrm{C}$ NMR $\left(100 \mathrm{MHz}\right.$, Methanol- $\left.d_{4}\right) \delta$ : $144.41,143.31$, 139.19, 134.49, 130.57, 129.89, 122.18, 121.40, 120.82, 120.55, 120.08, 114.41, 110.52, 39.87, 19.60. HRMS calcd for $\mathrm{C}_{22} \mathrm{H}_{20} \mathrm{~N}_{5}[\mathrm{M}+\mathrm{H}]^{+} 354.1713$, found 354.1703 .

1-Methyl-9-((1-(4-fluorophenyl)-1H-1,2,3-triazol-4-yl)methyl)- $\beta$-carboline (5k): Slightly brown crystals $(0.71$ $\mathrm{g}, 80 \%)$ were obtained. ${ }^{1} \mathrm{H}$ NMR $\left(400 \mathrm{MHz}, \mathrm{DMSO}-d_{6}\right) \delta: 8.82(\mathrm{~s}, 1 \mathrm{H}), 8.27-8.24(\mathrm{~m}, 2 \mathrm{H}), 8.03(\mathrm{~d}$, $J=5.2 \mathrm{~Hz}, 1 \mathrm{H}), 7.90-7.86(\mathrm{~m}, 3 \mathrm{H}), 7.63-7.58(\mathrm{~m}, 1 \mathrm{H}), 7.43-7.38(\mathrm{~m}, 2 \mathrm{H}), 7.29(\mathrm{t}, J=7.6 \mathrm{~Hz}, 1 \mathrm{H}), 6.00(\mathrm{~s}$, 2H), $3.13(\mathrm{~s}, 3 \mathrm{H}) .{ }^{13} \mathrm{C}$ NMR $\left(100 \mathrm{MHz}\right.$, DMSO-d $\left.d_{6}\right) \delta: 162.04(\mathrm{~d}, J=244 \mathrm{~Hz}), 145.48,142.33,141.41,138.34$, $135.09,133.43(\mathrm{~d}, J=2.6 \mathrm{~Hz}), 128.70,128.64,122.97,122.88,121.99(\mathrm{~d}, J=10 \mathrm{~Hz}), 121.33,120.35,117.11$ $(\mathrm{d}, J=23.1 \mathrm{~Hz}), 113.50,111.20,23.88$. HRMS calcd for $\mathrm{C}_{21} \mathrm{H}_{17} \mathrm{FN}_{5}[\mathrm{M}+\mathrm{H}]^{+} 358.1463$, found 358.1470 .

9-Butyl-1-methyl-7-((1-(4-(trifluoromethyl)phenyl)-1H-1,2,3-triazol-4-yl)methoxy)- $\beta$-carboline (9a): Slightly yellow crystals $(0.41 \mathrm{~g}, 85 \%)$ were obtained. ${ }^{1} \mathrm{H}$ NMR $\left(400 \mathrm{MHz}, \mathrm{DMSO}-d_{6}\right) \delta: 9.17(\mathrm{~s}, 1 \mathrm{H}), 8.21-8.17$ $(\mathrm{m}, 3 \mathrm{H}), 8.12(\mathrm{~d}, J=8.4 \mathrm{~Hz}, 1 \mathrm{H}), 8.01(\mathrm{~d}, J=8.4 \mathrm{~Hz}, 2 \mathrm{H}), 7.88(\mathrm{~d}, J=5.2 \mathrm{~Hz}, 1 \mathrm{H}), 7.40(\mathrm{~d}, J=2.4 \mathrm{~Hz}$, $1 \mathrm{H}), 6.98(\mathrm{dd}, J=8.8,2.0 \mathrm{~Hz}, 1 \mathrm{H}), 5.45(\mathrm{~s}, 2 \mathrm{H}), 4.56(\mathrm{t}, J=7.6 \mathrm{~Hz}, 2 \mathrm{H}), 2.95(\mathrm{~s}, 3 \mathrm{H}), 1.75-1.67(\mathrm{~m}, 2 \mathrm{H})$, $1.42-1.32(\mathrm{~m}, 2 \mathrm{H}), 0.89(\mathrm{t}, J=7.6 \mathrm{~Hz}, 3 \mathrm{H}) .{ }^{13} \mathrm{C}$ NMR $\left(100 \mathrm{MHz}, \mathrm{DMSO}-d_{6}\right) \delta: 159.49,144.80,143.10$, $141.07,139.80(\mathrm{q}, J=1.5 \mathrm{~Hz}), 138.22,135.10,129.40(\mathrm{q}, J=32.4 \mathrm{~Hz}), 128.79,127.73(\mathrm{q}, J=3.6 \mathrm{~Hz}), 124.27$ $(\mathrm{q}, J=270.5 \mathrm{~Hz}) 123.63,122.95,121.04,115.06,112.76,109.96,95.37,61.99,44.38,32.93,23.55,19.98$, 14.19. HRMS calcd for $\mathrm{C}_{26} \mathrm{H}_{25} \mathrm{~F}_{3} \mathrm{~N}_{5} \mathrm{O}[\mathrm{M}+\mathrm{H}]^{+} 480.2017$, found 480.2009 .

9-Butyl-1-methyl-7-((1-(3,4,5-trifluorophenyl)-1H-1,2,3-triazol-4-yl)methoxy)- $\beta$-carboline (9b): Slightly brown crystals $(0.43 \mathrm{~g}, 91 \%)$ were obtained. ${ }^{1} \mathrm{H}$ NMR $\left(400 \mathrm{MHz}, \mathrm{DMSO}-d_{6}\right) \delta: 8.76(\mathrm{~s}, 1 \mathrm{H}), 8.18-8.13(\mathrm{~m}$, $2 \mathrm{H}), 8.04(\mathrm{~s}, 1 \mathrm{H}), 7.86-7.82(\mathrm{~m}, 2 \mathrm{H}), 7.32(\mathrm{~s}, 1 \mathrm{H}), 7.07(\mathrm{~d}, J=8.8 \mathrm{~Hz}, 1 \mathrm{H}), 5.47(\mathrm{~s}, 2 \mathrm{H}), 4.63(\mathrm{t}, J=7.6 \mathrm{~Hz}$, $2 \mathrm{H}), 3.07(\mathrm{~s}, 3 \mathrm{H}), 1.87-1.79(\mathrm{~m}, 2 \mathrm{H}), 1.52-1.43(\mathrm{~m}, 2 \mathrm{H}), 1.00(\mathrm{t}, J=7.6 \mathrm{~Hz}, 3 \mathrm{H}) .{ }^{13} \mathrm{C} \mathrm{NMR}(100 \mathrm{MHz}$, DMSO- $\left.d_{6}\right) \delta: 159.54,152.34(\mathrm{ddd}, J=246.6,10.0,5.2 \mathrm{~Hz}), 144.86,143.17,140.91,138.70(\mathrm{dt}, J=250$, $18 \mathrm{~Hz}), 137.90,135.02,132.58(\mathrm{td}, J=12.1,3.6 \mathrm{~Hz}), 128.92,123.64,122.96,115.02,112.78,110.00,106.17$ (m), 95.29, 62.01, 44.38, 32.91, 23.32, 19.97, 14.16. HRMS calcd for $\mathrm{C}_{25} \mathrm{H}_{23} \mathrm{~F}_{3} \mathrm{~N}_{5} \mathrm{O}[\mathrm{M}+\mathrm{H}]^{+} 466.1849$, found 466.1860 .

9-Butyl-1-methyl-7-((1-(4-(trifluoromethoxy)phenyl)-1H-1,2,3-triazol-4-yl)methoxy)- $\beta$-carboline (9c): Yellow crystals $(0.44 \mathrm{~g}, 88 \%)$ were obtained. ${ }^{1} \mathrm{H}$ NMR $\left(400 \mathrm{MHz}\right.$, DMSO- $\left.d_{6}\right) \delta: 9.10(\mathrm{~s}, 1 \mathrm{H}), 8.17(\mathrm{~d}, J=5.2 \mathrm{~Hz}$, $1 \mathrm{H}), 8.15-8.04(\mathrm{~m}, 3 \mathrm{H}), 7.89(\mathrm{~d}, J=5.2 \mathrm{~Hz}, 1 \mathrm{H}), 7.65(\mathrm{~d}, J=8.0 \mathrm{~Hz}, 2 \mathrm{H}), 7.41(\mathrm{~d}, J=2.0 \mathrm{~Hz}, 1 \mathrm{H}), 6.97(\mathrm{dd}$, $J=8.4,2.0 \mathrm{~Hz}, 1 \mathrm{H}), 5.43(\mathrm{~s}, 2 \mathrm{H}), 4.58(\mathrm{t}, J=7.6 \mathrm{~Hz}, 2 \mathrm{H}), 2.95(\mathrm{~s}, 3 \mathrm{H}), 1.74-1.66(\mathrm{~m}, 2 \mathrm{H}), 1.41-1.32(\mathrm{~m}$, $2 \mathrm{H}), 0.89(\mathrm{t}, J=7.6 \mathrm{~Hz}, 3 \mathrm{H}) .{ }^{13} \mathrm{C}$ NMR $\left(100 \mathrm{MHz}, \mathrm{DMSO}-d_{6}\right) \delta: 159.46,148.34,144.55,143.11,141.05$, 138.20, 135.87, 128.79, 123.77, 123.11, 122.95, 122.57, 121.73, 119.17, 114.97, 112.77, 110.06, 95.35, 61.87, 44.34, 32.96, 23.52, 19.93, 14.18. HRMS calcd for $\mathrm{C}_{26} \mathrm{H}_{25} \mathrm{~F}_{3} \mathrm{~N}_{5} \mathrm{O}_{2}[\mathrm{M}+\mathrm{H}]^{+} 496.1955$, found 496.1962 .

9-Butyl-7-((1-(4-methoxyphenyl)-1H-1,2,3-triazol-4-yl)methoxy)-1-methyl- $\beta$-carboline (9d): White crystals $(0.39 \mathrm{~g}, 89 \%)$ were obtained. ${ }^{1} \mathrm{H}$ NMR $\left(400 \mathrm{MHz}, \mathrm{DMSO}-d_{6}\right) \delta: 8.55(\mathrm{~s}, 1 \mathrm{H}), 8.04(\mathrm{~d}, J=8.4 \mathrm{~Hz}, 1 \mathrm{H}), 7.89$ $(\mathrm{s}, 1 \mathrm{H}), 7.76-7.69(\mathrm{~m}, 2 \mathrm{H}), 7.20(\mathrm{~d}, J=2.0 \mathrm{~Hz}, 1 \mathrm{H}), 7.13-7.05(\mathrm{~m}, 2 \mathrm{H}), 7.00(\mathrm{dd}, J=8.4,2.0 \mathrm{~Hz}, 1 \mathrm{H}), 5.40$ $(\mathrm{s}, 2 \mathrm{H}), 4.53(\mathrm{t}, J=7.6 \mathrm{~Hz}, 2 \mathrm{H}), 3.87(\mathrm{~s}, 3 \mathrm{H}), 2.97(\mathrm{~s}, 3 \mathrm{H}), 1.80-1.72(\mathrm{~m}, 2 \mathrm{H}), 1.47-1.37(\mathrm{~m}, 2 \mathrm{H}), 0.96(\mathrm{t}$, $J=7.2 \mathrm{~Hz}, 3 \mathrm{H}) .{ }^{13} \mathrm{C}$ NMR $\left(100 \mathrm{MHz}\right.$, DMSO- $\left.d_{6}\right) \delta: 159.77,159.56,144.10,143.12,141.08,138.16,130.45$, 128.80, 123.35, 122.91, 122.22, 115.35, 114.97, 112.78, 109.99, 95.31, 62.02, 56.02, 44.36, 32.94, 23.57, 19.97, 14.20. HRMS calcd for $\mathrm{C}_{26} \mathrm{H}_{28} \mathrm{~N}_{5} \mathrm{O}_{2}[\mathrm{M}+\mathrm{H}]^{+} 442.2238$, found 442.2247.

9-Butyl-1-methyl-7-((1-(p-tolyl)-1H-1,2,3-triazol-4-yl)methoxy)- $\beta$-carboline (9e): Slightly yellow crystals $(0.36 \mathrm{~g}, 86 \%)$ were obtained. ${ }^{1} \mathrm{H}$ NMR $\left(400 \mathrm{MHz}, \mathrm{DMSO}-d_{6}\right) \delta: 9.06(\mathrm{~s}, 1 \mathrm{H}), 8.52(\mathrm{~d}, J=6.0 \mathrm{~Hz}, 1 \mathrm{H})$, $8.41(\mathrm{~d}, J=8.8 \mathrm{~Hz}, 1 \mathrm{H}), 8.35(\mathrm{~d}, J=6.4 \mathrm{~Hz}, 1 \mathrm{H}), 7.81(\mathrm{~d}, J=8.4 \mathrm{~Hz}, 2 \mathrm{H}), 7.63(\mathrm{~d}, J=2.0 \mathrm{~Hz}, 1 \mathrm{H}), 7.42(\mathrm{~d}$, $J=8.4 \mathrm{~Hz}, 2 \mathrm{H}), 7.14(\mathrm{dd}, J=8.8,2.0 \mathrm{~Hz}, 1 \mathrm{H}), 5.49(\mathrm{~s}, 2 \mathrm{H}), 4.69(\mathrm{t}, J=8.0 \mathrm{~Hz}, 2 \mathrm{H}), 3.26(\mathrm{~s}, 3 \mathrm{H}), 2.39(\mathrm{~s}$, $3 \mathrm{H}), 1.82-1.74(\mathrm{~m}, 2 \mathrm{H}), 1.45-1.35(\mathrm{~m}, 2 \mathrm{H}), 0.91(\mathrm{t}, J=7.2 \mathrm{~Hz}, 3 \mathrm{H}) .{ }^{13} \mathrm{C}$ NMR $\left(100 \mathrm{MHz}, \mathrm{DMSO}-d_{6}\right) \delta$ : $161.94,146.31,143.74,138.92,137.71,134.76,133.82,133.18,130.74,129.45,125.00,123.56,120.48,114.86$, 
$113.71,113.15,95.24,62.27,44.83,32.86,21.06,19.89,17.93,14.17$. HRMS calcd for $\mathrm{C}_{26} \mathrm{H}_{28} \mathrm{~N}_{5} \mathrm{O}[\mathrm{M}+\mathrm{H}]^{+}$ 426.2288, found 426.2281 .

9-Butyl-7-((1-(4-fluorophenyl)-1H-1,2,3-triazol-4-yl)methoxy)-1-methyl- $\beta$-carboline (9f): White crystals (041 $\mathrm{g}, 87 \%)$ were obtained. ${ }^{1} \mathrm{H}$ NMR $\left(400 \mathrm{MHz}, \mathrm{DMSO}-d_{6}\right) \delta: 9.03(\mathrm{~s}, 1 \mathrm{H}), 8.18(\mathrm{~d}, J=5.2 \mathrm{~Hz}, 1 \mathrm{H}), 8.12(\mathrm{~d}$, $J=8.8 \mathrm{~Hz}, 1 \mathrm{H}), 8.03-7.96(\mathrm{~m}, 2 \mathrm{H}), 7.89(\mathrm{~d}, J=5.2 \mathrm{~Hz}, 1 \mathrm{H}), 7.48(\mathrm{t}, J=8.8 \mathrm{~Hz}, 2 \mathrm{H}), 7.41(\mathrm{~d}, J=2.0 \mathrm{~Hz}$, $1 \mathrm{H}), 6.98(\mathrm{dd}, J=8.8,2.0 \mathrm{~Hz}, 1 \mathrm{H}), 5.43(\mathrm{~s}, 2 \mathrm{H}), 4.56(\mathrm{t}, J=7.6 \mathrm{~Hz}, 2 \mathrm{H}), 2.95(\mathrm{~s}, 3 \mathrm{H}), 1.75-1.67(\mathrm{~m}, 2 \mathrm{H})$, $1.42-1.33(\mathrm{~m}, 2 \mathrm{H}), 0.90(\mathrm{t}, J=7.6 \mathrm{~Hz}, 3 \mathrm{H}) .{ }^{13} \mathrm{C}$ NMR $\left(100 \mathrm{MHz}, \mathrm{DMSO}-d_{6}\right) \delta: 160.91(\mathrm{~d}, J=245 \mathrm{~Hz})$, $159.52,144.39,143.11,141.05,138.20,135.08,133.61(\mathrm{~d}, J=2.8 \mathrm{~Hz}), 128.80,123.64,122.98(\mathrm{~d}, J=8.7 \mathrm{~Hz})$, $122.93,117.22(\mathrm{~d}, J=23.3 \mathrm{~Hz}), 115.00,112.75,109.99,95.33,61.98,44.36,32.94,23.54,19.96,14.20$. HRMS calcd for $\mathrm{C}_{25} \mathrm{H}_{25} \mathrm{FN}_{5} \mathrm{O}[\mathrm{M}+\mathrm{H}]^{+} 430.2038$, found 430.2044 .

\subsection{Biological Assays}

The antifungal activity of the synthesized compounds was performed according to previously reported procedures [33]. The fungicidal activity of the target compounds against $R$. solani, F. oxysporum, B. cinerea Pers., sunflower sclerotinia rot and rape sclerotinia rot were evaluated using a mycelium growth rate test [17]. Carbendazim and azoxystrobin standard purchased from J\&K Scientific Ltd. (Beijing, China), were used as a control, treating it in the same way. The relative inhibition ratio (\%) was calculated using the following equation:

The relative inhibition ratio $(\%)=\frac{\text { Colony diameter of control }- \text { colony diameter of treated })}{\text { colony diameter of control mycelial disk diameter }} \times 100 \%$.

\section{Conclusions}

In order to find potential activity from $\beta$-carboline derivatives for further structural optimization, in this study, two series of new aryl-1,2,3-triazole- $\beta$-carboline hybrids were synthesized, and first assayed for their fungicidal activities in vitro. The antifungal evaluation of the novel hybrids showed that, among the tested compounds, 1 -methyl-9-((1-phenyl-1H-1,2,3-triazol-4-yl)methyl)- $\beta$-carboline (5a), 1-methyl-9-((1-(4-(trifluoromethyl)phenyl)-1 $H$-1,2,3-triazol-4-yl)methyl)- $\beta$-carboline (5b), 1-methyl-9-((1-(3,4,5-trifluorophenyl)-1 $H$-1,2,3-triazol-4-yl)methyl)- $\beta$-carboline (5c), and 9-butyl1-methyl-7-((1-(3,4,5-trifluorophenyl)-1H-1,2,3-triazol-4-yl)methoxy)- $\beta$-carboline $\quad(9 \mathbf{b})$ showed satisfactory antifungal activity against sunflower sclerotinia rot. Specifically, compound $\mathbf{9 b}$ also exhibited high broad-spectrum fungicidal activity against all the tested fungi with inhibition rates of $58.3 \%, 18.52 \%, 63.07 \%, 84.47 \%$, and $81.23 \%$. However, for F. oxysporum, all the target compounds showed no in vitro antifungal activities with an inhibition rate lower than $20 \%$.

Supplementary Materials: The following are available online, ${ }^{1} \mathrm{H}$ and ${ }^{13} \mathrm{C}$ NMR spectra for the target compounds are available online.

Author Contributions: J.Z., X.-Q.H. and B.D. conceived and designed the research; X.-Y.H., L.G. and X.-F.C. performed the experiments; Y.-T.Z. performed the bioassay research; X.-Y.H. and X.-Q.H. analyzed the data; J.Z. and L.G. wrote the paper.

Funding: This research was supported by the scientific research innovation project in Xinjiang Uygur Autonomous Region under Grant (XJGRI2017045); the Program for Changjiang Scholars and Innovative Research Team in University (No. IRT15R46), and Yangtze River Scholar Research Project of Shihezi University (No. CJXZ201601).

Conflicts of Interest: The authors declare no conflict of interest.

\section{References}

1. Dayan, F.E.; Cantrell, C.L.; Duke, S.O. Natural products in crop protection. Bioorg. Med. Chem. 2009, 17, 4022-4034. [CrossRef] [PubMed]

2. Rosell, G.; Quero, C.; Coll, J.; Guerrero, A. Biorational insecticides in pest management. J. Pestic. Sci. 2008, 33, 103-121. [CrossRef] 
3. Petroski, R.J.; Stanley, D.W. Natural compounds for pest and weed control. J. Agric. Food Chem. 2009, 57, 8171-8179. [CrossRef] [PubMed]

4. Crombie, L. Natural product chemistry and its part in the defence against insects and fungi in agriculture. Pestic. Sci. 1999, 55, 761-774. [CrossRef]

5. Meester, C.D. Genotoxic potential of $\beta$-carbolines: A review. Mutat. Res. 1995, 339, 139-153. [CrossRef]

6. Cain, M.; Weber, R.W.; Guzman, F.; Cook, J.M.; Barker, S.A.; Rice, K.C.; Crawley, J.N.; Paul, S.M.; Skolnick, P. $\beta$-Carbolines: Synthesis and neurochemical and pharmacological actions on brain benzodiazepine receptors. J. Med. Chem. 1982, 25, 1081-1091. [CrossRef] [PubMed]

7. Bournine, L.; Bensalem, S.; Fatmi, S.; Bedjou, F.; Mathieu, V.; Iguer-Ouada, M.; Kiss, R.; Duez, P. Evaluation of the cytotoxic and cytostatic activities of alkaloid extracts from different parts of Peganum harmala $\mathrm{L}$. (Zygophyllaceae). Eur. J. Integr. Med. 2017, 9, 91-96. [CrossRef]

8. Asgarpanah, J.; Ramezanloo, F. Chemistry, pharmacology and medicinal properties of Peganum harmala L. Afr. J. Pharm. Pharmacol. 2012, 6, 1573-1580. [CrossRef]

9. Srivastava, S.K.; Agarwal, A.; Chauhan, P.M.S.; Agarwal, S.K.; Bhaduri, A.P.; Singh, S.N.; Fatima, N.; Chatterjee, R.K. Potent 1,3-disubstituted-9H-pyrido[3,4-b] indoles as new lead compounds in antifilarial chemotherapy. Bioorg. Med. Chem. 1999, 7, 1223-1236. [CrossRef]

10. Wang, Y.H.; Tang, J.G.; Wang, R.R.; Yang, L.M.; Dong, Z.J.; Du, L.; Shen, X.; Liu, J.K.; Zheng, Y.T. Flazinamide, a novel $\beta$-carboline compound with anti-HIV actions. Biochem. Biophys. Res. Commun. 2007, 355, 1091-1095. [CrossRef] [PubMed]

11. Zhang, Z.J.; Zhang, J.J.; Jiang, Z.Y.; Zhong, G.H. Design, Synthesis and Bioactivity Evaluation of Novel $\beta$-carboline 1,3,4-oxadiazole Derivatives. Molecules 2017, 22, 1811. [CrossRef] [PubMed]

12. Nenaah, G. Toxicity and growth inhibitory activities of methanol extract and the beta-carboline alkaloids of Peganum harmala L. against two coleopteran stored-grain pests. J. Stored Prod. Res. 2011, 47, 255-261. [CrossRef]

13. Abbasipour, H.; Mahmoudvand, M.; Rastegar, F.; Basij, M. Insecticidal activity of Peganum harmala seed extract against the diamondback moth, Plutella xylostella. Bull. Insectol. 2010, 63, 259-263.

14. Shonouda, M.; Osman, S.; Salama, O.; Ayoub, A. Toxical effect of Peganum harmala L. leaves on the cotton leaf worm, Spodoptera littoralis boisd and its parasitoids Microplitis rufiventris Kok. Pak. J. Biol. Sci. 2008, 11, 546-552. [CrossRef] [PubMed]

15. Song, H.J.; Liu, Y.X.; Liu, Y.X.; Wang, Q.M. Synthesis and antiviral and fungicidal activity evaluation of $\beta$-carboline, dihydro- $\beta$-carboline, tetrahydro- $\beta$-carboline alkaloids, and their derivatives. J. Agric. Food Chem. 2014, 62, 1010-1018. [CrossRef] [PubMed]

16. Huang, Y.Q.; Liu, Y.X.; Liu, Y.X.; Song, H.J.; Wang, Q.M. C ring may be dispensable for $\beta$-carboline: Design, synthesis, and bioactivities evaluation of tryptophan analog derivatives based on the biosynthesis of $\beta$-carboline alkaloids. Bioorg. Med. Chem. 2016, 24, 462-473. [CrossRef] [PubMed]

17. Li, Z.B.; Chen, S.H.; Zhu, S.W.; Luo, J.J.; Zhang, Y.M.; Weng, Q.F. Synthesis and fungicidal activity of $\beta$-carboline alkaloids and their derivatives. Molecules 2015, 20, 13941-13957. [CrossRef] [PubMed]

18. Huo, X.Y.; Guo, L.; Wei, Y.T.; Zhang, J.; Han, X.Q. Synthesis and Fungicidal Activity of Novel $\beta$-Carboline Derivatives. Agrochemicals 2018, 57, 3-6. [CrossRef]

19. Ruddarraju, R.R.; Murugulla, A.C.; Kotla, R.; Tirumalasetty, M.C.B.; Wudayagiri, R.; Donthabakthuni, S.; Maroju, R.; Baburao, K.; Parasa, L.S. Design, synthesis, anticancer, antimicrobial activities and molecular docking studies of theophylline containing acetylenes and theophylline containing 1,2,3-triazoles with variant nucleoside derivatives. Eur. J. Med. Chem. 2016, 123, 379-396. [CrossRef] [PubMed]

20. Kant, R.; Kumar, D.; Agarwal, D.; Gupta, R.D.; Tilak, R.; Awasthi, S.K.; Agarwal, A. Synthesis of newer 1,2,3-triazole linked chalcone and flavone hybrid compounds and evaluation of their antimicrobial and cytotoxic activities. Eur. J. Med. Chem. 2016, 113, 34-49. [CrossRef] [PubMed]

21. Huo, J.P.; Hu, H.W.; Zhang, M.; Hu, X.H.; Chen, M.; Chen, D.C.; Liu, J.W.; Xiao, G.F.; Wang, Y.; Wen, Z.L. A mini review of the synthesis of poly-1,2,3-triazole-based functional materials. RSC Adv. 2017, 7, 2281-2287. [CrossRef]

22. Floros, M.C.; Bortolatto, J.F.; Oliveira, J.O.B.; Salvador, S.L.; Narine, S.S. Antimicrobial activity of amphiphilic triazole-linked polymers derived from renewable sources. ACS Biomater. Sci. Eng. 2016, 2, 336-343. [CrossRef] 
23. Vatmurge, N.S.; Hazra, B.G.; Pore, V.S.; Shirazi, F.; Chavan, P.S.; Dehpande, M.V. Synthesis and antimicrobial activity of $\beta$-lactam-bile acid conjugates linked via triazole. Bioorg. Med. Chem. Lett. 2008, 18, 2043-2047. [CrossRef] [PubMed]

24. Jagasia, R.; Holub, J.M.; Bollinger, M.; Kirshenbaum, K.; Finn, M.G. Peptide Cyclization and Cyclodimerization by $\mathrm{Cu}^{\mathrm{I}}$-Mediated Azide-Alkyne Cycloaddition. J. Org. Chem. 2009, 74, 2964-2974. [CrossRef] [PubMed]

25. Huber, D.; Hübner, H.; Gmeiner, P. 1,1'-Disubstituted Ferrocenes as Molecular Hinges in Mono- and Bivalent Dopamine Receptor Ligands. J. Med. Chem. 2009, 52, 6860-6870. [CrossRef] [PubMed]

26. Dubovis, M.V.; Rudakov, G.F.; Kulagin, A.S.; Tsarkova, K.V.; Popkov, S.V.; Goloveshkin, A.S.; Cherkaev, G.V. A new method of synthesis of substituted 1-(1H-imidazole-4-yl)-1H-1,2,3-triazoles and their fungicidal activity. Tetrahedron 2018, 74, 672-683. [CrossRef]

27. Zheng, X.C.; Wan, Y.J.; Ling, F.; Ma, C. Copper-Catalyzed Tandem Reaction of Terminal Alkynes and Sulfonyl Azides for the Assembly of Substituted Aminotriazoles. Org. Lett. 2017, 19, 3859-3862. [CrossRef] [PubMed]

28. Hao, C.B.; Zhou, C.J.; Xie, J.W.; Zhang, J.; Liu, P.; Dai, B. An Efficient Copper-Catalyzed One-Pot Synthesis of 1-Aryl-1,2,3-triazoles from Arylboronic Acids in Water under Mild Conditions. Chin. J. Chem. 2015, 33, 1317-1320. [CrossRef]

29. Zhou, C.J.; Zhang, J.; Liu, P.; Xie, J.W.; Dai, B. 2-Pyrrolecarbaldiminato-Cu(II) complex catalyzed three-component 1,3-dipolar cycloaddition for 1,4-disubstituted 1,2,3-triazoles synthesis in water at room temperature. RSC Adv. 2015, 5, 6661-6665. [CrossRef]

30. Cao, R.H.; Chen, Q.; Hou, X.R.; Chen, H.S.; Guan, H.J.; Ma, Y.; Peng, W.L.; Xu, A.L. Synthesis, acute toxicities and antitumor effects of novel 9-substituted $\beta$-carboline derivatives. Bioorg. Med. Chem. 2004, 12, 4613-4623. [CrossRef] [PubMed]

31. Xinjiang Huashidan Pharmaceutical Research Co., Ltd. Harmine Derivatives, Intermediates Used in Their Preparation, Preparation Processes and Use Thereof. EP 1634881 A1, 15 March 2006.

32. Cao, R.H.; Guan, X.D.; Shi, B.X.; Chen, Z.Y.; Ren, Z.H.; Peng, W.L.; Song, H.C. Design, synthesis and 3D-QSAR of $\beta$-carboline derivatives as potent antitumor agents. Eur. J. Med. Chem. 2010, 45, 2503-2515. [CrossRef] [PubMed]

33. Du, S.J.; Lu, H.Z.; Yang, D.Y.; Li, H.; Gu, X.L.; Wan, C.; Jia, C.Q.; Wang, M.; Li, X.Y.; Qin, Z.H. Synthesis, Antifungal Activity and QSAR of Some Novel Carboxylic Acid Amides. Molecules 2015, 20, 4071-4087. [CrossRef] [PubMed]

Sample Availability: Samples of the compounds $\mathbf{5 a}-\mathbf{k}, \mathbf{9 a}-\mathbf{f}$ are available from the authors.

(C) 2018 by the authors. Licensee MDPI, Basel, Switzerland. This article is an open access article distributed under the terms and conditions of the Creative Commons Attribution (CC BY) license (http:/ / creativecommons.org/licenses/by/4.0/). 